Research Article

\title{
Effects of Fermented Wheat Bran on Flour, Dough, and Steamed Bread Characteristics
}

\author{
Li Li, ${ }^{1,2}$ Zhen Wang, ${ }^{2}$ Li-Min $\mathrm{Li}^{2}{ }^{2}$ Xue-Ling Zheng $\mathbb{D}^{2},{ }^{2}$ Sen Ma $\mathbb{D}^{1,2}$ and Xiao-Xi Wang ${ }^{2}$ \\ ${ }^{1}$ National Engineering Laboratory for Wheat \& Corn Further Processing, Henan University of Technology, \\ Zhengzhou 450001, China \\ ${ }^{2}$ College of Grain Oil and Food Science, Henan University of Technology, Zhengzhou 450001, China \\ Correspondence should be addressed to Xue-Ling Zheng; xlzhenghaut@126.com and Sen Ma; masen@haut.edu.cn
}

Received 31 August 2017; Revised 13 December 2017; Accepted 12 April 2018; Published 19 June 2018

Academic Editor: Yanjie Bai

Copyright (C) $2018 \mathrm{Li} \mathrm{Li} \mathrm{et} \mathrm{al.} \mathrm{This} \mathrm{is} \mathrm{an} \mathrm{open} \mathrm{access} \mathrm{article} \mathrm{distributed} \mathrm{under} \mathrm{the} \mathrm{Creative} \mathrm{Commons} \mathrm{Attribution} \mathrm{License,} \mathrm{which}$ permits unrestricted use, distribution, and reproduction in any medium, provided the original work is properly cited.

\begin{abstract}
Wheat bran is rich in dietary fiber, which improves the flour nutritional content and also endows the flour with a richer flavor. However, a high content of insoluble dietary bran fiber can easily and significantly reduce the processing and edible quality of flour products. This study was conducted to explore ways to decrease the negative effects of adding bran to dough. Basidiomycete strain BS-01 was used to ferment the wheat bran. The surface structure of the bran was examined by scanning electron microscopy, and the fermented bran was incorporated into the wheat flour at various concentrations. The mixed flour farinographic and extensographic characteristics, dough rheological properties, and the specific volume, color, and textural properties of the steamed bread were determined and analyzed. The results suggested that adding an appropriate quantity of fermented bran improves the characteristics of the dough and the quality of the steamed bread compared to those with unfermented bran. The fermented bran effectively decreased the negative impacts exerted on the farinographic and extensographic characteristics of the mixed flour and exerted a positive influence on the dough viscoelasticity and bread specific volume.
\end{abstract}

\section{Introduction}

Wheat bran is the outer layer of the wheat kernel and an excellent source of dietary fiber (DF), which contains several nutrients, such as starch, protein, fat, minerals, and vitamins [1]. It has a wide range of applications in the modern food industry; for example, many popular foods of cereal origin, such as noodles, breads, and cookies, are good carriers of wheat bran DF [2]. However, due to the fibrous external layer, adding wheat bran can decrease the product sensory quality [3].

The content of DF in wheat bran is $35-50 \%$, whereas the content of soluble DF is only 2-3\% [4]. However, for wheat bran DF to be considered as a high-quality DF supplement, it requires a soluble DF content $>10 \%$; otherwise, it is only considered as filling DF [5]. Thus, many researchers have been dedicated to modify wheat bran DF so as to improve the soluble DF content in wheat bran. Nevertheless, there is still a lack of studies on the impact of insoluble DF on food eating quality. Moreover, insoluble DF impairs gas cell stabilization while making flour products, which reduces the products quality [6]. Adding raw wheat bran has a detrimental effect on the rheological properties of dough and steamed bread quality, dough stretching energy, elongation, and tensile resistance. For instance, the maximum tensile resistance decreases gradually as the amount of wheat bran added increases. Also, the hardness, gumminess, and chewiness of steamed bread increase gradually, whereas resilience, cohesiveness, and recovery decrease $[7,8]$.

To improve the eating quality and nutrition, many researchers have focused on modifying the properties of wheat bran, and several microorganisms have been adopted for fermentation [9-11]. Fermented bran increases the content and bioavailability of several functional compounds, such as water-extractable arabinoxylans, total free phenols, and soluble fiber, but only has a slight impact on physical properties. As a result, when forming the dough, the $\mathrm{pH}$ and 
total acidity values increase, and the maximum height, gas retention, and stabilization time of the dough decrease after adding wheat bran fermented by Lactobacillus [12]. Even after processing by complex microbial agents (yeast, Lactobacillus, and the sweet wine koji), dough extensibility and the texture of steamed bread become worse after mixing with fermented bran [13]. These properties make it very difficult to improve the functional quality of flour products by mixing in wheat bran.

The insoluble DF content includes up to $90 \%$ hemicellulose and lignin [4]. Lignin fiber is strongly chemically resistant because of the conserved units, complex structure, random chemical bonding, and high molecular weight. Lignin and hemicellulose are linked by covalent bonds in the wheat bran and form a dense structure with cellulose embedded in the structure [14]. This solid structure prevents the use of common methods to sufficiently modify wheat bran and greatly reduces the nutritional value and eating quality of wheat bran and the food products containing it [15]. The soluble DF content in edible wheat bran can be increased, and the quality and nutrition can be effectively improved by targeted removal of the structural barrier formed by lignin and hemicellulose and disassembly of the interspace reticular structure. Accordingly, the Basidiomycetes which have been proved to exhibit a wide range of lignin and hemicellulose degradation activities become the optimal choice in wheat bran DF modification [16].

In this study, the Basidiomycete strain BS-01 was used to ferment wheat bran. Scanning electron microscopy (SEM) analysis was used to evaluate the effect of fermentation on the bran surface structure. In addition, the fermented bran was incorporated at various concentrations into wheat flour and steamed bread. Then, the flour farinographic and extensographic characteristics, dough rheological properties, technological qualities, and the correlations between them were determined. Additionally, the specific volume, color, and textural properties of bran mixed with steamed bread were also evaluated. This study may stimulate further interest in the use of fermentation to modify edible wheat bran.

\section{Materials and Methods}

2.1. Materials. Wheat flour (11.59\% moisture, $0.59 \%$ ash, and $12.02 \%$ protein) was obtained from ZhengZhou TDR Flour Industrial Co., Ltd. (Zhengzhou, China). Wheat bran was obtained from Guangdong Baiyan Grain \& Oil Industrial Co., Ltd. (Foshan, China). Yeast powder was purchased from Angel Yeast Co. (Yichang, China). BS-01 (CGMCC 3.7572) was obtained from the China General Microbiological Culture Collection Center (Beijing, China). $\alpha$-Amylase A4551 $(10 \mathrm{U} / \mathrm{mg})$ and protease K P-6556 (30 U/mg) were purchased from Sigma-Aldrich Co. (St. Louis, MO, USA). All other chemical reagents were purchased from Luoyang Chemical Reagents Factory and used directly.

2.2. Stock Culture Conditions. The stock culture was maintained on potato dextrose agar (PDA) slants at $4^{\circ} \mathrm{C}$ with periodic transfer. The PDA medium contained $200 \mathrm{~g}$ potato infusion, $20 \mathrm{~g}$ glucose, $3 \mathrm{~g} \mathrm{KH}_{2} \mathrm{PO}_{4}, 1.5 \mathrm{~g} \cdot \mathrm{MgSO}_{4}$, and $15 \mathrm{~g}$ agar per liter. The mycelium from the slant was transferred to PDA plates and incubated at $28^{\circ} \mathrm{C}$ for 7 days to produce the inoculum. Five mycelial discs $(5 \mathrm{~mm}$ diameter $)$ were removed from the peripheral region of the PDA plates and used to inoculate Erlenmeyer flasks $(250 \mathrm{~mL})$ containing $50 \mathrm{~mL}$ of liquid potato dextrose (PD) medium. The cultures were incubated at $28^{\circ} \mathrm{C}$ on a rotary shaker incubator at $160 \mathrm{rpm} / \mathrm{min}$ [17].

2.3. Bran Fermentation. The solid culture medium contained $2 \mathrm{~g}$ sugar, $2 \mathrm{~g} \mathrm{CaSO}_{4} \cdot 2 \mathrm{H}_{2} \mathrm{O}$, and $196 \mathrm{~g}$ raw wheat bran per $200 \mathrm{~g}$ [18]. The moisture content of the medium was adjusted to $55 \mathrm{~mL} / 100 \mathrm{~g}$, and the medium was loaded into an autoclavable gusseted breathable plastic bulk mushroom grow bag. The loaded bags were prepared in triplicate and autoclaved at $121^{\circ} \mathrm{C}$ and 15 psi for $120 \mathrm{~min}$. After cooling the bags to room temperature, they were inoculated with $2 \mathrm{ml}$ of the BS-01 spore suspension and then incubated under standard conditions $\left(22 \pm 2^{\circ} \mathrm{C}\right.$ and $60 \%$ relative humidity) for 10 days. The raw and fermented wheat bran was dried in a vacuum drier at $60^{\circ} \mathrm{C}$ until the moisture content was $3-4 \%$ and then pulverized and filtered through a 100-mesh sieve, and the screen residue were collected for SEM analysis.

2.4. Scanning Electron Microscopic Analysis. SEM was used to evaluate the effect of fermentation on wheat bran characteristics. The bran samples were suspended in $250 \mathrm{~mL}$ of $0.5 \mathrm{M}$ sodium phosphate buffer ( $\mathrm{pH}$ 6.5), and $\alpha$-amylase was added to $3 \%$ and incubated in a water bath at $60^{\circ} \mathrm{C}$ for $1 \mathrm{~h}$. Then, the $\mathrm{pH}$ value of the suspension was adjusted to 4.5 , protease was added to in $3 \%$, and the samples were incubated in a water bath at $60^{\circ} \mathrm{C}$ for $2 \mathrm{~h}$. After the enzyme treatment, the samples were rinsed three times with the same buffer and dehydrated (twice in each solution) through a graded ethanol series $(20,40,60,80,95$, and $100 \%)$ for $10 \mathrm{~min}$ at each concentration. After drying to constant weight $\left(48^{\circ} \mathrm{C}\right.$ for $72 \mathrm{~h}$ ), the samples were sputter-coated with palladium gold in the Emitech K550 and observed under a Zeiss DSM 940 A SEM system (Carl Zeiss, Oberkochen, Germany) at 2,000x magnification.

2.5. Steamed Bread Preparation. Steamed bread was prepared according to the sponge dough method described by Song et al. [19] with some modifications. Batches of $100 \mathrm{~g}$ of mixed flour (14.0\% moisture base mixed with $0,3,6,9$, and $12 \%$ fermented or unfermented wheat bran powder by weight), $1 \mathrm{~g}$ active dry yeast, and water were mixed at low speed for 10 min using a flour mixer (JHMZ200, East Fude Technology Development Center, Beijing, China). Water content was about $85 \%$ of the water absorption of flour. Dough was leavened in a fermenting box for $1 \mathrm{~h}$ at $38^{\circ} \mathrm{C}$ and $85 \%$ relative humidity after mixing. The dough was sheeted 15 times at $3.5 \mathrm{~mm}$ (YMG110, Jiangsu Hengyue Co. Ltd., Jiangsu, China), rolled into a long cylindrical shape by hand, and divided into pieces of a specified weight (100 g pieces) and round in shape. Ultimately, the dough pieces were steamed 

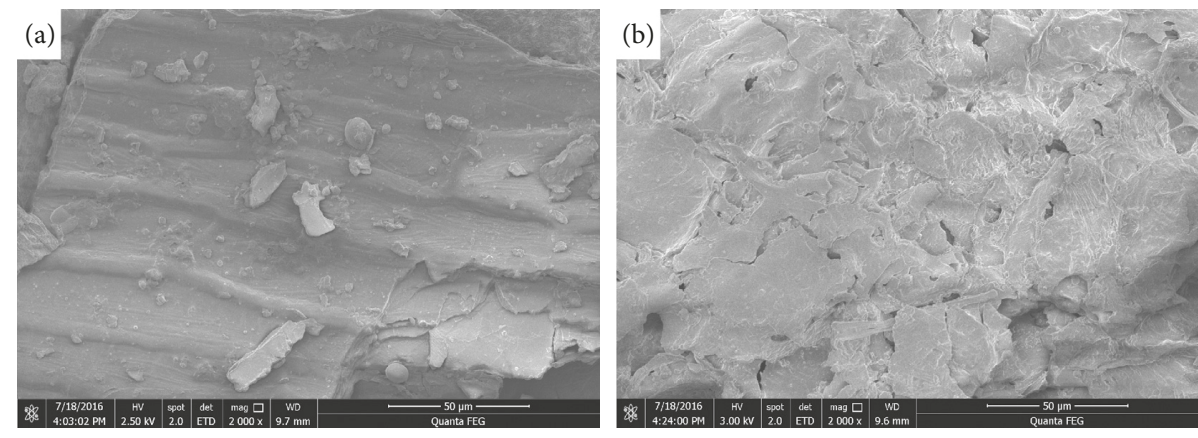

FIGURE 1: Scanning electron microscopic images of the surface structure of wheat bran: (a) unfermented wheat bran and (b) fermented wheat bran.

for $30 \mathrm{~min}$ in a steamer and boiling water (WSYH26A, Midea Co., Ltd., Guangdong, China). The steamed bread was covered with gauze and cooled at room temperature for $60 \mathrm{~min}$ before the quality evaluation. Each dough treatment was performed in duplicate.

2.6. Farinographic and Extensographic Properties. The rheological properties of the flour samples of nonfermented dough (flour water system) were measured with a farinograph (Farinograph-E, Brabender, GmbH \& Co. KG, Duisburg, Germany) and an extensograph (Brabender Extensograph-E) following the AACC 54-21 and 54-10 standard methods.

2.7. Rheological Measurement. The dynamic rheological properties of the dough samples were determined with a rotational rheometer (MARSIII, Haake, Vreden, Germany) using the measuring system plate-plate (HPP 20) following the method of Stojceska et al. [20].

2.8. Steamed Bread Volume and Color Measurements. The bread volume, expressed as $\mathrm{mL} / \mathrm{g}$, was determined according to AACC Method 10-05 (AACC, 2000). Color values were measured with a Minolta CM-508i spectrophotometer (Minolta Co. Ltd., Tokyo, Japan) using the D65 standard illuminant and the $2^{\circ}$ standard observer.

2.9. Steamed Bread Texture Measurements. The textural analysis was performed with a Texture analyzer (Model: TAXT2i, Texture Technologies Corp., Scarsdale, NY, USA), equipped with a $25 \mathrm{~mm}$ diameter aluminum cylindrical probe. The steamed bread was sliced horizontally, and a bottom piece of $25 \mathrm{~mm}$ in height was compressed to $50 \%$ of the original height. Test conditions were as follows: pretest speed $3 \mathrm{~mm} / \mathrm{s}$, test speed $1 \mathrm{~mm} / \mathrm{s}$, and posttest speed $5 \mathrm{~mm} / \mathrm{s}$. The analysis was performed in triplicate.

2.10. Statistical Analysis. For all analyses, two technical replicates were used, and the data were statistically compared by analysis of variance (ANOVA). The significant differences between two samples were analyzed by Duncan's test using the SPSS software (version 15.0, SPSS Inc., Chicago, IL, USA). A $p$ value $<0.05$ was considered significant.

\section{Results and Discussion}

3.1. SEM Microstructure Analysis. SEM was used to examine the effect of fermentation on the surface microstructure of wheat bran.

The microstructure of the surface of two bran samples is shown in Figure 1. Specifically, raw wheat bran (Figure 1(a)) had a compact, smooth surface structure with many sharp edges. In comparison, due to the effective decomposition of the bran fiber by fermentation, the surface of the fermented bran (Figure 1(b)) was porous and rugged, with the Basidiomycete mycelia occupying the interspaces between the bran cells and concentrated in the microholes of the bran surface. The bran cell walls were broken down from the lumens to a compound middle lamella, while the bran cellulose, hemicellulose, and lignin were degraded by microbial metabolites. After fermentation, the porous surface structure was favorable to improve the rehydration and water absorption capacity of the bran mixed products. Moreover, the unsharpened edges helped reduce the negative effect of bran on the gas-holding capacity of the dough.

\subsection{Farinograph Characterization of Bran Mixed Flour.} The farinograph dough development time (DDT) represents the formation speed of the gluten network after wheat flour is combined with water [21]. The results listed in Table 1 show that the DDT was constantly prolonged as the proportion of wheat bran increased, but the DDT of the dough mixed with fermented bran was shorter than that of raw bran in most cases.

The dough stability time (ST), breakdown time (BT), and the quality index (QI) were used to assess the mixing tolerance of the bran mixed flour [22]. The protein concentration in the mixed flour was lower when the bran content was higher, which prevented formation of a good viscoelastic gluten network. The results show that the ST, BT, and the three QIs of the fermented bran mixed flour were highest when the bran content was 3\% and then decreased gradually. The rate of decrease of the mixed fermented bran sample was slower than that of the unfermented bran. The softening degree (SD) of the mixed flour dough increased correspondingly with the increase of the bran content, and the extent of the increase was 
TABLE 1: Different amounts of added bran and the farinographic properties of the mixed flour.

\begin{tabular}{|c|c|c|c|c|c|c|}
\hline Added amount & Bran state & DDT (min) & ST (min) & $\mathrm{SD}(\mathrm{FU})$ & $\mathrm{BT}(\min )$ & QI \\
\hline $0 \%$ & & $5.2^{\mathrm{b}}$ & $7.6^{\mathrm{a}}$ & $76^{\mathrm{d}}$ & $9.5^{\mathrm{a}}$ & $95^{\mathrm{a}}$ \\
\hline \multirow{2}{*}{$3 \%$} & Fermented & $6.1^{\mathrm{a}}$ & $7.9^{\mathrm{a}}$ & $89^{c}$ & $10.0^{\mathrm{a}}$ & $100^{\mathrm{a}}$ \\
\hline & Raw & $4.8^{\mathrm{b}}$ & $7.1^{\mathrm{a}}$ & $79^{\mathrm{d}}$ & $9.2^{\mathrm{a}}$ & $92^{\mathrm{a}}$ \\
\hline \multirow{2}{*}{$6 \%$} & Fermented & $4.1^{b}$ & $7.5^{\mathrm{a}}$ & $102^{\mathrm{b}}$ & $8.9^{\mathrm{a}}$ & $89^{\mathrm{a}}$ \\
\hline & Raw & $6.0^{\mathrm{a}}$ & $6.7^{\mathrm{b}}$ & $95^{\mathrm{c}}$ & $8.9^{\mathrm{a}}$ & $89^{\mathrm{a}}$ \\
\hline \multirow{2}{*}{$9 \%$} & Fermented & $5.4^{\mathrm{b}}$ & $6.9^{\mathrm{a}}$ & $116^{\mathrm{a}}$ & $8.6^{\mathrm{b}}$ & $86^{\mathrm{b}}$ \\
\hline & Raw & $5.7^{\mathrm{a}}$ & $5.8^{\mathrm{b}}$ & $105^{\mathrm{b}}$ & $8.5^{\mathrm{b}}$ & $85^{\mathrm{b}}$ \\
\hline \multirow{2}{*}{$12 \%$} & Fermented & $6.8^{\mathrm{a}}$ & $6.9^{\mathrm{a}}$ & $123^{\mathrm{a}}$ & $9.1^{\mathrm{a}}$ & $91^{\mathrm{a}}$ \\
\hline & Raw & $6.6^{\mathrm{a}}$ & $5.7^{\mathrm{b}}$ & $110^{\mathrm{b}}$ & $8.7^{\mathrm{b}}$ & $87^{\mathrm{b}}$ \\
\hline
\end{tabular}

Values for a particular column followed by different letters differ significantly $(p<0.05)$. DDT, dough development time; ST, stability time; SD, softening degree; BT, breakdown time; QI, qualitative index.

TABLe 2: Different amounts of added bran and the extensographic properties of the mixed flour.

\begin{tabular}{|c|c|c|c|c|c|c|c|}
\hline Added amount & Bran state & $\mathrm{EE}\left(\mathrm{cm}^{2}\right)$ & $\mathrm{ES}(\mathrm{mm})$ & $\mathrm{RE}(\mathrm{FU})$ & MR (FU) & SR & Maximum SR \\
\hline $0 \%$ & & $70.5^{\mathrm{a}}$ & $147.0^{\mathrm{a}}$ & $301.0^{\mathrm{b}}$ & $371.0^{\mathrm{a}}$ & $2.10^{\mathrm{b}}$ & $2.55^{\mathrm{e}}$ \\
\hline \multirow{2}{*}{$3 \%$} & Fermented & $73.0^{\mathrm{a}}$ & $125.0^{\mathrm{a}}$ & $355.5^{\mathrm{a}}$ & $416.5^{\mathrm{a}}$ & $3.00^{\mathrm{b}}$ & $3.35^{\mathrm{a}}$ \\
\hline & Raw & $58.5^{\mathrm{b}}$ & $115.0^{\mathrm{b}}$ & $341.5^{\mathrm{a}}$ & $370.0^{\mathrm{a}}$ & $3.00^{\mathrm{b}}$ & $3.25^{\mathrm{b}}$ \\
\hline \multirow{2}{*}{$6 \%$} & Fermented & $58.0^{\mathrm{b}}$ & $114.0^{\mathrm{b}}$ & $340.0^{\mathrm{a}}$ & $358.0^{\mathrm{a}}$ & $2.80^{\mathrm{b}}$ & $3.20^{\mathrm{c}}$ \\
\hline & Raw & $51.5^{\mathrm{c}}$ & $104.5^{\mathrm{c}}$ & $339.0^{\mathrm{b}}$ & $355.5^{\mathrm{a}}$ & $3.25^{\mathrm{a}}$ & $3.40^{\mathrm{a}}$ \\
\hline \multirow[b]{2}{*}{$9 \%$} & Fermented & $46.5^{\mathrm{c}}$ & $94.5^{\mathrm{c}}$ & $349.0^{\mathrm{a}}$ & $352.5^{\mathrm{a}}$ & $3.70^{\mathrm{a}}$ & $3.70^{\mathrm{a}}$ \\
\hline & Raw & $45.0^{\mathrm{d}}$ & $97.0^{\mathrm{C}}$ & $331.5^{\mathrm{b}}$ & $341.5^{\mathrm{b}}$ & $3.45^{\mathrm{a}}$ & $3.50^{\mathrm{a}}$ \\
\hline \multirow{2}{*}{$12 \%$} & Fermented & $39.5^{\mathrm{d}}$ & $93.0^{\mathrm{C}}$ & $291.0^{\mathrm{b}}$ & $292.0^{\mathrm{b}}$ & $3.10^{\mathrm{b}}$ & $3.10^{\mathrm{c}}$ \\
\hline & Raw & $42.5^{\mathrm{d}}$ & $96.5^{\mathrm{c}}$ & $319.5^{\mathrm{b}}$ & $322.5^{\mathrm{b}}$ & $3.35^{\mathrm{a}}$ & $3.35^{\mathrm{a}}$ \\
\hline
\end{tabular}

Values for a particular column followed by different letters differ significantly $(p<0.05)$. EE, extension energy; ES, extensibility; RE, resistance to extension; $\mathrm{MR}$, maximum resistance; SR, stretching ratio.

more evident when the mixed bran was fermented. Taken together, the farinograph properties of the fermented bran mixed flour were relatively lower than those of the unfermented flour with equal amounts of added bran.

\subsection{Extensographic Characterization of Bran Mixed Flour.} Resistance to extension (RE) indicates the strength of the gluten network and gas-holding capacity of the dough [23]. The results shown in Table 2 indicate that adding more than $3 \%$ bran caused a gradual decline in the RE. However, the RE and maximum resistance (MR) values of the fermented bran mixed flour were higher than those in the unfermented group, when the amount of bran added was $<12 \%$.

Extensibility (ES) is indicative of the ductility and plasticity of the dough, and the extension energy (EE) provides information about the strength of the gluten and the baking properties of the flour [24]. The results show that although the ES and EE decreased steadily with the increase of the amount of bran added, but when the amount added was $<12 \%$, both two indices are relatively higher in the fermented sample than in the unfermented one under the same bran added amount.

The dough stretching ratio is the maximum resistance to the extensibility ratio. The results in this study show that the stretching ratio of the bran mixed dough increased steadily, as unfermented bran was added, while the ratio of the fermented sample initially increased and then decreased. This extensographic properties analysis showed that adding fermented bran enhanced the strength of the dough network and had a better effect on the dough than unfermented bran with less bran was added.

3.4. Rheological Characterization of Bran Mixed Dough. The dough viscoelasticity represents the structural strength and degree of combination with water and has important effects on the flour product making process [25]. The average values of the elastic modulus $\left(G^{\prime}\right)$, viscous modulus $\left(G^{\prime \prime}\right)$, and $\tan \delta\left(G^{\prime \prime} / G^{\prime}\right)$ for the fermented and unfermented bran mixed dough are shown in Figures 2-4.

The data presented in Figures 2 and 3 reveal that when the same amount of bran was added, the $G^{\prime}$ and $G^{\prime \prime}$ values of the fermented samples were higher than those of the unfermented samples, and both values were relatively higher, when the amount of bran added was $6 \%$ or $12 \%$. This indicates that adding fermented bran favors the formation of the gluten network and enhances dough viscoelasticity. Moreover, the $G^{\prime}$ and $G^{\prime \prime}$ values of the $9 \%$ fermented bran samples were all higher than those of the $3 \%$ samples, demonstrating that adding fermented bran improved the gas-holding capacity of the dough. However, similar trends in the $G^{\prime}$ and $G^{\prime \prime}$ values were not observed for the unfermented groups.

The $\tan \delta$ indicates the relative contributions of the viscous $\left(G^{\prime \prime}\right)$ and elastic $\left(G^{\prime}\right)$ characteristics of the material. When the material behaves more like a solid, that is, when deformation within the linear range is essentially elastic and recoverable, the elastic character, $G^{\prime}$, exceeds the viscous character, $G^{\prime \prime}$, and $\tan \delta<1$. On the other hand, when the material behaves more like a liquid or viscous system, then 


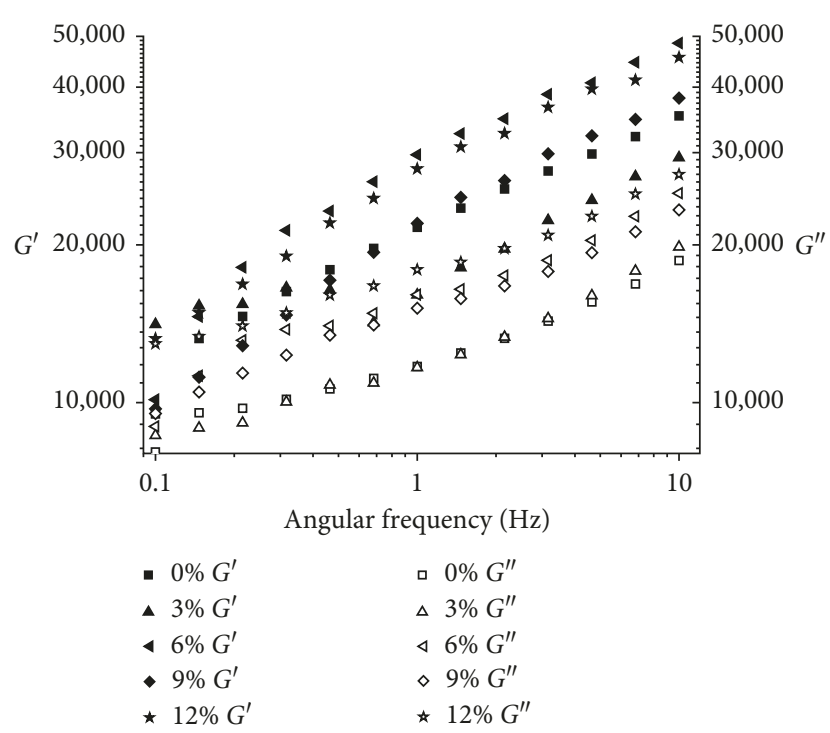

Figure 2: The effect of fermented bran addition on the $G^{\prime}$ and $G^{\prime \prime}$ of bread dough.

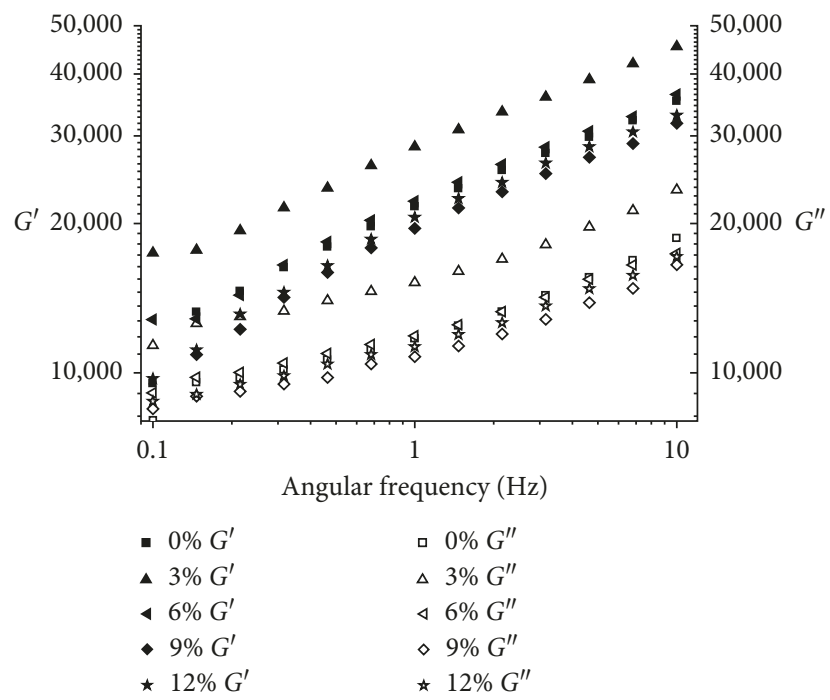

FIgURE 3: The effect of unfermented bran addition on the $G^{\prime}$ and $G^{\prime \prime}$ of bread dough.

the viscous character, $G^{\prime \prime}$, predominates and $\tan \delta>1$ [26]. The results presented in Figure 4 indicate that the $\tan \delta$ value of the bran mixed dough gradually decreased as the amount of the unfermented bran was increased, and the $\tan \delta$ value of the fermented bran mixed dough changed from randomly scattered to consistent with the increase of the shear rate. This probably occurred because the fermentation changed the physical structure and chemical composition of the bran, and these complex physical and chemical changes led to the irregular shifts in the dough viscoelasticity.

3.5. Bread Volume, Color, and Texture of Bran Mixed Flour. The specific volume is an important indicator used to estimate gas-holding capacity of steamed bread dough. Wheat bran

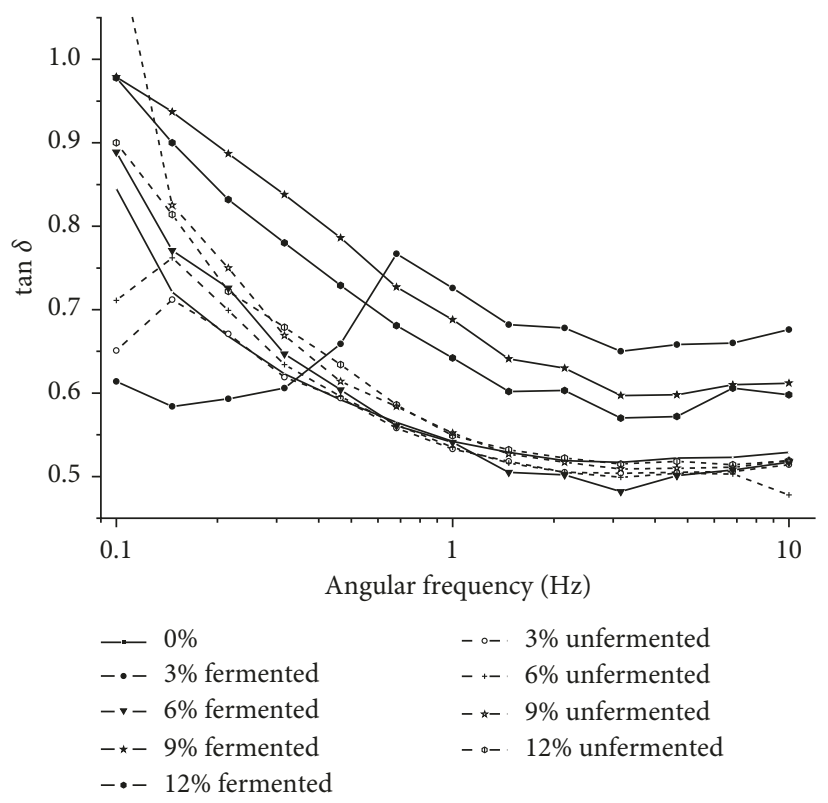

Figure 4: The effect of fermented and unfermented bran addition on the $\tan \delta$ of bread dough.

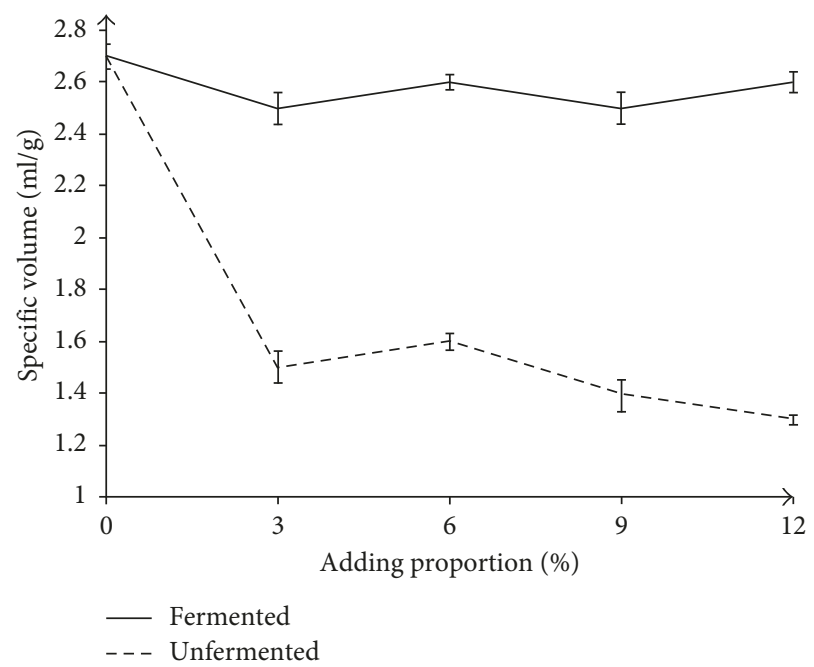

FIGURE 5: The effect of bran addition on the specific volume of steamed bread.

contains a high raw fiber content, which can damage the gluten network and break the gas-holding cells in the dough. Accordingly, adding bran likely affects the specific volume negatively, as confirmed by the results shown in Figure 5.

The specific volume of steamed bread initially increased and then decreased as the amount of unfermented bran added increased (Figure 5). The increase was steady when fermented bran was used and was more than twice that of the unfermented bran mixed sample with the same amount of added bran. This finding indicates that fermentation diminished the negative effect of adding bran on the quality of steamed bread.

The color values of steamed bread were recorded as $L^{*}$ (0, black; 100, white), $a^{*}(-100$, green; +100 , red $)$, and 


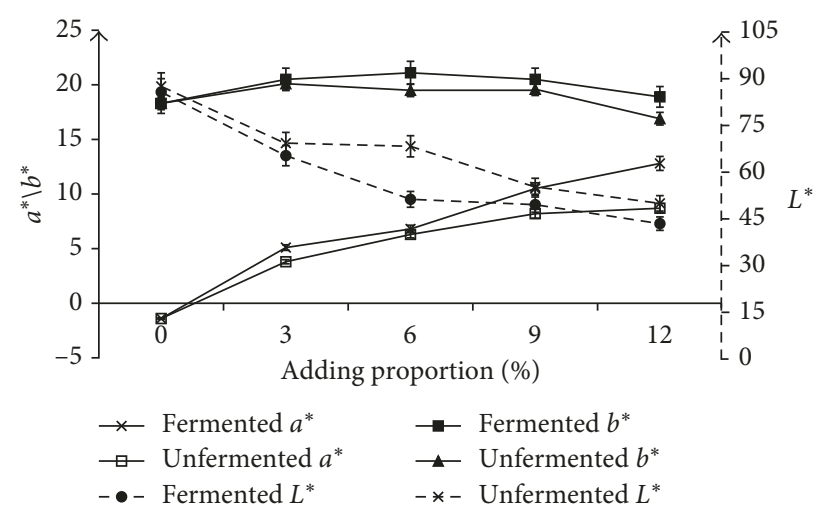

Figure 6: The effect of bran addition on the color of steamed bread.

TABle 3: Different amounts of added bran and the texture of mixed steamed bread.

\begin{tabular}{|c|c|c|c|c|c|c|c|}
\hline Addition amount & Bran state & Hardness (g) & Resilience & Cohesiveness & Springiness & Adhesiveness & Chewiness \\
\hline $0 \%$ & & $2528.89^{\mathrm{b}}$ & $41.19^{\mathrm{a}}$ & $0.76^{\mathrm{a}}$ & $94.28^{\mathrm{a}}$ & $1921.77^{\mathrm{b}}$ & $1811.13^{\mathrm{b}}$ \\
\hline \multirow{2}{*}{$3 \%$} & Fermented & $11775.18^{\mathrm{a}}$ & $32.29^{\mathrm{b}}$ & $0.67^{\mathrm{a}}$ & $88.17^{\mathrm{b}}$ & $7875.12^{\mathrm{a}}$ & $6967.19^{\mathrm{a}}$ \\
\hline & Raw & $3359.18^{\mathrm{b}}$ & $40.67^{\mathrm{a}}$ & $0.77^{\mathrm{a}}$ & $90.33^{\mathrm{a}}$ & $2594.86^{\mathrm{b}}$ & $2344.12^{\mathrm{b}}$ \\
\hline \multirow{2}{*}{$6 \%$} & Fermented & $17017.31^{\mathrm{a}}$ & 29.68 & 0.61 & $85.98^{\mathrm{b}}$ & $10326.57^{\mathrm{a}}$ & $8880.28^{\mathrm{a}}$ \\
\hline & Raw & $5432.35^{\mathrm{b}}$ & $42.90^{\mathrm{a}}$ & $0.82^{\mathrm{a}}$ & $91.96^{\mathrm{a}}$ & $4445.68^{\mathrm{a}}$ & $4089.49^{a}$ \\
\hline \multirow[b]{2}{*}{$9 \%$} & Fermented & $13627.58^{\mathrm{a}}$ & 26.74 & $0.58^{\mathrm{b}}$ & $80.03^{\mathrm{b}}$ & $7938.69^{a}$ & $6347.52^{\mathrm{a}}$ \\
\hline & Raw & $5244.33^{\mathrm{b}}$ & $41.31^{\mathrm{a}}$ & $0.79^{\mathrm{a}}$ & $88.28^{\mathrm{b}}$ & $4143.02^{\mathrm{b}}$ & $3659.05^{\mathrm{a}}$ \\
\hline \multirow{2}{*}{$12 \%$} & Fermented & $10350.28^{\mathrm{a}}$ & $31.65^{\mathrm{b}}$ & $0.64^{\mathrm{b}}$ & 49.81 & $6165.72^{\mathrm{a}}$ & $3716.70^{\mathrm{a}}$ \\
\hline & Raw & $4183.14^{\mathrm{b}}$ & $41.01^{\mathrm{a}}$ & $0.79^{\mathrm{a}}$ & $87.59^{\mathrm{b}}$ & $3323.32^{\mathrm{b}}$ & $2910.51^{\mathrm{b}}$ \\
\hline
\end{tabular}

Values for a particular column followed by different letters differ significantly $(p<0.05)$.

$b^{*}\left(-100\right.$, blue; +100 , yellow). As shown in Figure 6 , the $L^{*}$ value of steamed bread steadily decreased as the amount of bran added was increased and significantly decreased in the fermented bran mixed group. The $a^{*}$ and $b^{*}$ values of steamed bread increased with the increase of the amount of bran added. All fermented bran mixed sample values were relatively higher, but within acceptable limits. Fermentation deepened the color of the wheat bran.

The hardness, adhesiveness, and chewiness values represent the chewy texture of steamed bread, and higher values indicate a worse texture. On the other hand, the springiness, cohesiveness, and resilience represent the chewing quality of steamed bread, and higher values mean chewier bread [27]. The results listed in Table 3 show that bran had a strong effect on the textural quality of steamed bread, regardless of bran fermentation. The hardness, adhesiveness, and chewiness values increased as the amount of bran added increased, whereas springiness, cohesiveness, and resilience significantly decreased, due to a decline in the bread specific volume and water-absorbing capacity of gluten caused by the addition of fiber-rich bran.

The increases in bread hardness, adhesiveness, and chewiness and the decreases in springiness, cohesiveness, and resilience were sharper as the amount of fermented bran added increased, compared with the unfermented bran mixed samples. The changing tendencies of the hardness, adhesiveness, and chewiness of steamed bread were similar. Bread resilience improved slightly when the amount of fermented bran added was $\leq 9 \%$.

\section{Conclusion}

The results obtained in this study suggest that adding fermented wheat bran improved the characteristics of the dough and improved some quality characteristics of steamed bread compared to unfermented bran. Fermentation by the BS-01 strain modified the bran structure, efficiently decreased the negative effects on the farinographic and extensographic characteristics of the mixed flour caused by the addition of bran, and exerted a positive influence on the dough viscoelasticity and bread specific volume. These results indicate that modifying wheat bran by fermentation is an effective method to improve the quality of high fiber flour products.

\section{Conflicts of Interest}

The authors declare that they have no conflicts of interest.

\section{Acknowledgments}

The present research was financially supported by National Natural Science Foundation of China (nos. 31671892 and U1704118), National College Students Innovation and Entrepreneurship Training Program Project (201710463002), Opening Foundation of National Engineering Laboratory for Wheat \& Corn Further Processing in Henan University of Technology (NL2016004), and Foundation Research 
Funds for the Henan Provincial Colleges and Universities in Henan University of Technology (2016QNJH18).

\section{References}

[1] L. Mosharraf, "Effect of processing on the decrease in phytic acid and pesticide residue in wheat bran," Journal of Agricultural Engineering Research, vol. 16, no. 2, pp. 71-82, 2015.

[2] A. Sobota, Z. Rzedzicki, P. Zarzycki, and E. Kuzawińska, "Application of common wheat bran for the industrial production of high-fibre pasta," International Journal of Food Science and Technology, vol. 50, no. 1, pp. 111-119, 2015.

[3] O. P. Lehtinen, Modifying Wheat Bran for Food ApplicationsEffect of Wet Milling and Enzymatic Treatment, Metropolia University of Applied Sciences, Helsinki, Finland, 2012.

[4] P. B. Schwarz, W. H. Kunerth, and V. L. Youngs, "The distribution of lignin and other fiber components within hard red spring wheat bran," Cereal Chemistry, vol. 65, no. 1, pp. 59-64, 1988.

[5] R. E. A. Leitz and D. J. Pusateri, "Balanced fiber composition," US Patent 4,877,627, 1989.

[6] S. S. Arya and S. K. Sonawane, "Impact of fiber mixture on dough and chapatti quality using D-optimal response surface methodology," Journal of Microbiology, Biotechnology and Food Sciences, vol. 5, no. 5, pp. 424-433, 2016.

[7] C. Liu, L. Liu, L. Li et al., "Effects of different milling processes on whole wheat flour quality and performance in steamed bread making," LWT-Food Science and Technology, vol. 62, no. 1, pp. 310-318, 2015.

[8] D. Zhang and W. R. Moore, "Effect of wheat bran particle size on dough rheological properties," Journal of the Science of Food and Agriculture, vol. 74, no. 4, pp. 490-496, 2015.

[9] K. Roopesh, S. Ramachandran, K. M. Nampoothiri, G. Szakacs, and A. Pandey, "Comparison of phytase production on wheat bran and oilcakes in solid-state fermentation by Mucor racemosus," Bioresource Technology, vol. 97, no. 3, pp. 506-511, 2006.

[10] H. Watanabe and M. Tadokoro, "Study of a two-stage fermentation process producing hydrogen and methane from wheat bran," in Proceedings of the Annual Conference of the Japan Society of Waste Management Experts, p. 169, Osaka, Japan 2007.

[11] M. C. Messia, A. Reale, L. Maiuro, T. Candigliota, E. Sorrentino, and E. Marconi, "Effects of pre-fermented wheat bran on dough and bread characteristics," Journal of Cereal Science, vol. 69, pp. 138-144, 2016.

[12] J. Basa, Naveena, M. Altaf, K. Bhadrayya, and G. Reddy, "Production of L (+) lactic acid by Lactobacillus amylophilus GV6 in semi-solid state fermentation using wheat bran," Food Technology and Biotechnology, vol. 472, no. 42, pp. 147-152, 2004.

[13] L. Xiong, X. Z. Cao, K. B. Lv, P. Gao, R. Ren, and T. Wu, "Effect of multi-strain fermentation on the quality of wheat bran steamed bread," Jiangsu Agricultural Sciences, vol. 41, no. 1, pp. 262-264, 2013.

[14] N. E. Anderson and F. M. C. Ydesdale, "An analysis of the dietary fiber, content of a standard wheat bran," Journal of Food Science, vol. 45, no. 2, pp. 336-340, 1980.

[15] I. Banu, G. Stoenescu, V. S. Ionescu, and I. Aprodu, "Effect of the addition of wheat bran stream on dough rheology and bread quality," Annals of the University Dunarea De Jos of Galati Fascicle VI Food Technology, vol. 36, no. 1, pp. 39-52, 2012.
[16] P. Mervana, S. P. Singh, and B. Vyas, "Solid state fermentation of wheat straw by white rot Basidiomycetes," in Proceedings of the UGC-CAS National Conference on Biodiversity and Bioresource Utilization, pp. 84-87, India, March 2015.

[17] G. Ginalska, H. Y. Cho, N. S. Cho et al., "Effect of culture conditions on growth and lipase production by a newly isolated strain, geotrichum-like R59 (Basidiomycetes)," Journal of the Faculty of Agriculture Kyushu University, vol. 52, no. 1, pp. 29-34, 2007.

[18] M. Y. Lung, "In vitro antioxidant properties of the edible Basidiomycete Phellinus igniarius by solid-state fermentation," in Proceedings of the Asia Pacific Confederation of Chemical Engineering Congress, pp. 862-863, Singapore, February 2012.

[19] H. E. Song, C. H. Liu, and J. J. Zuo, "Study on the technics of one-time-fermentation-method steamed-bread making procession," Grain Processing, vol. 4, no. 1, pp. 47-52, 2004.

[20] V. Stojceska, F. Butler, E. Gallagher, and D. Keehan, "A comparison of the ability of several small and large deformation rheological measurements of wheat dough to predict baking behaviour," Journal of Food Engineering, vol. 83 , no. 4 , pp. $475-482,2008$.

[21] C. Wang and M. I. P. Kovacs, "Swelling index of glutenin test. II. Application in prediction of dough properties and end-use quality," Cereal Chemistry, vol. 79, no. 2, pp. 190-196, 2007.

[22] B. L. D'Appolonia and W. H. Kunerth, Farinograph Handbook, American Association of Cereal Chemists Inc., St. Paul, MN, USA, 1984.

[23] K. R. Preston, The Extensograph Handbook, American Association of Cereal Chemists Inc., St. Paul, MN, USA, 1991.

[24] K. Wang, B. Dupuis, R. D. Cuthbert, and B. X. Fu, "A rapid extensigraph protocol for measuring dough viscoelasticity and mixing requirement," Journal of Cereal Science, vol. 76, pp. 99-107, 2017.

[25] S. Sofou, E. B. Muliawan, S. G. Hatzikiriakos, and E. Mitsoulis, "Rheological characterization and constitutive modeling of bread dough," Rheologica Acta, vol. 47, no. 4, pp. 369-381, 2008.

[26] W. F. Sollars, "Fractionation and reconstitution techniques for studying water-retention properties of wheat flours," Cereal Chemistry, vol. 50, no. 1, pp. 708-716, 1973.

[27] G. D. Valle, H. Chiron, L. Cicerelli et al., "Basic knowledge models for the design of bread texture," Trends in Food Science and Technology, vol. 36, no. 1, pp. 5-14, 2014. 

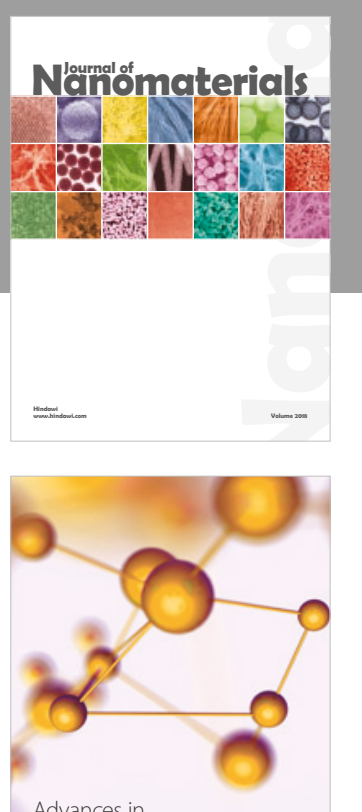

Physical Chemistry
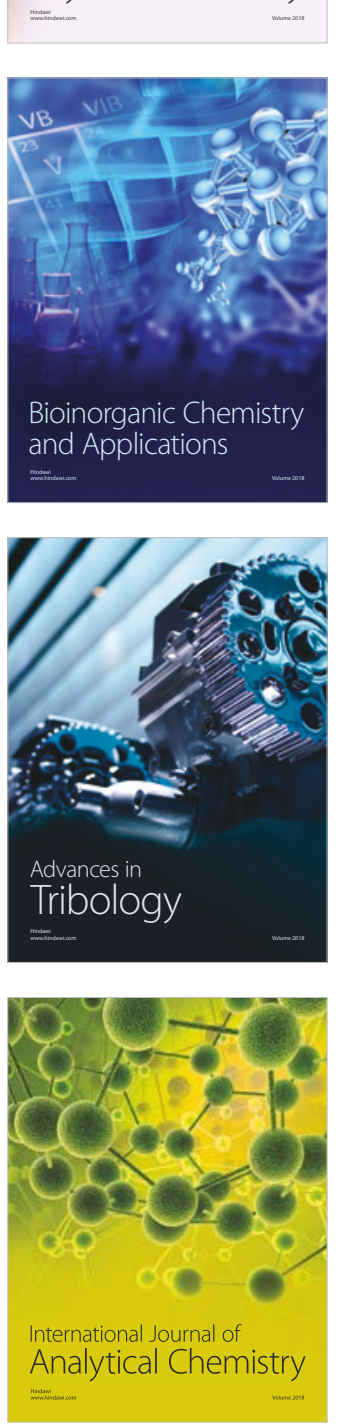

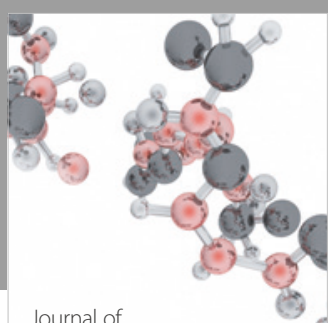

Analytical Methods

in Chemistry

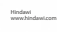

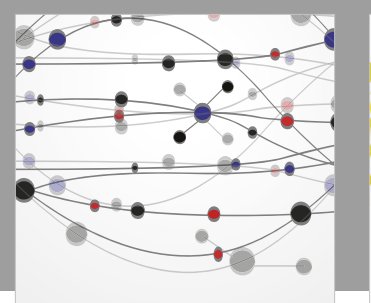

The Scientific World Journal

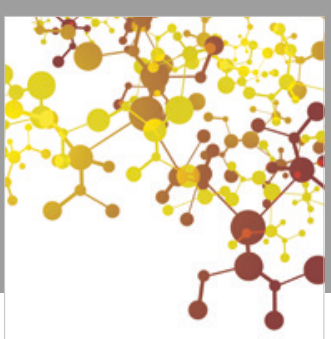

Journal of

Applied Chemistry
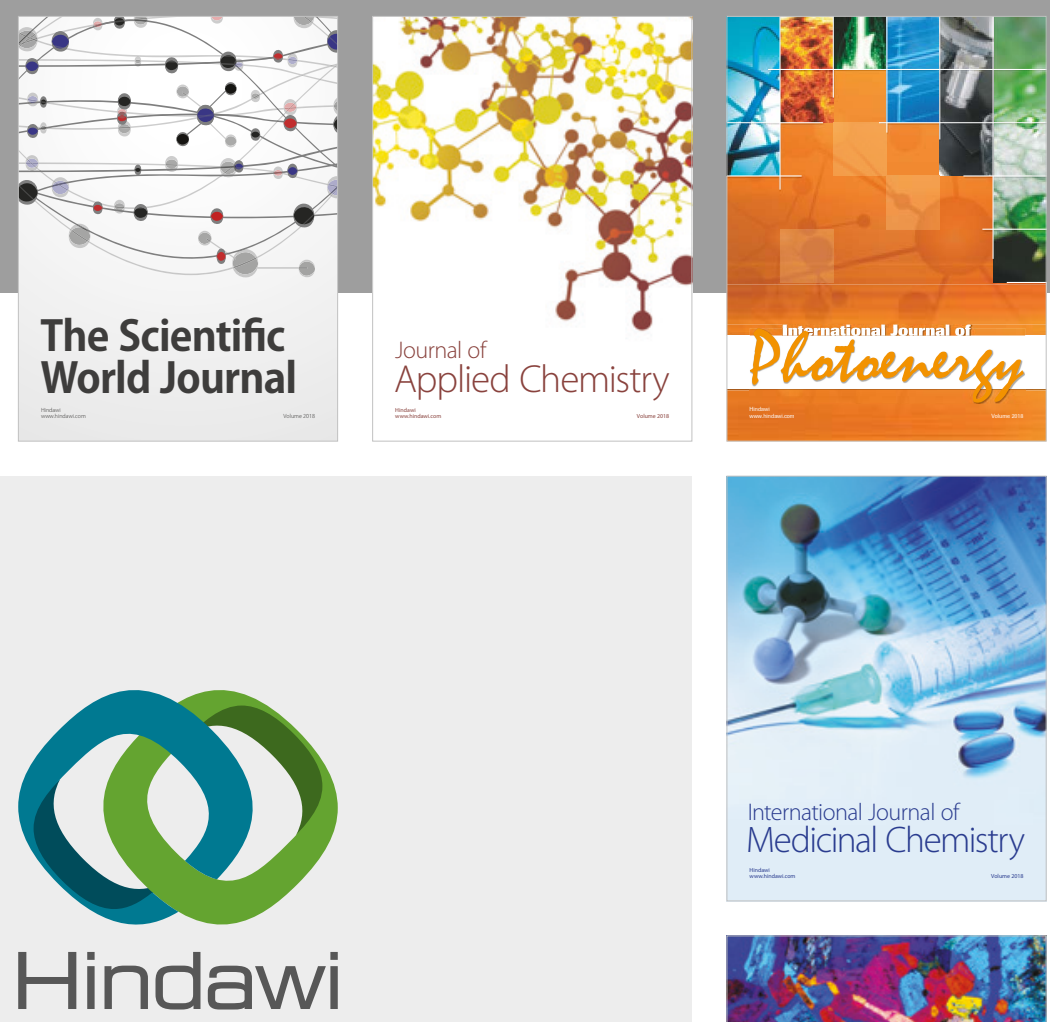

Submit your manuscripts at

www.hindawi.com
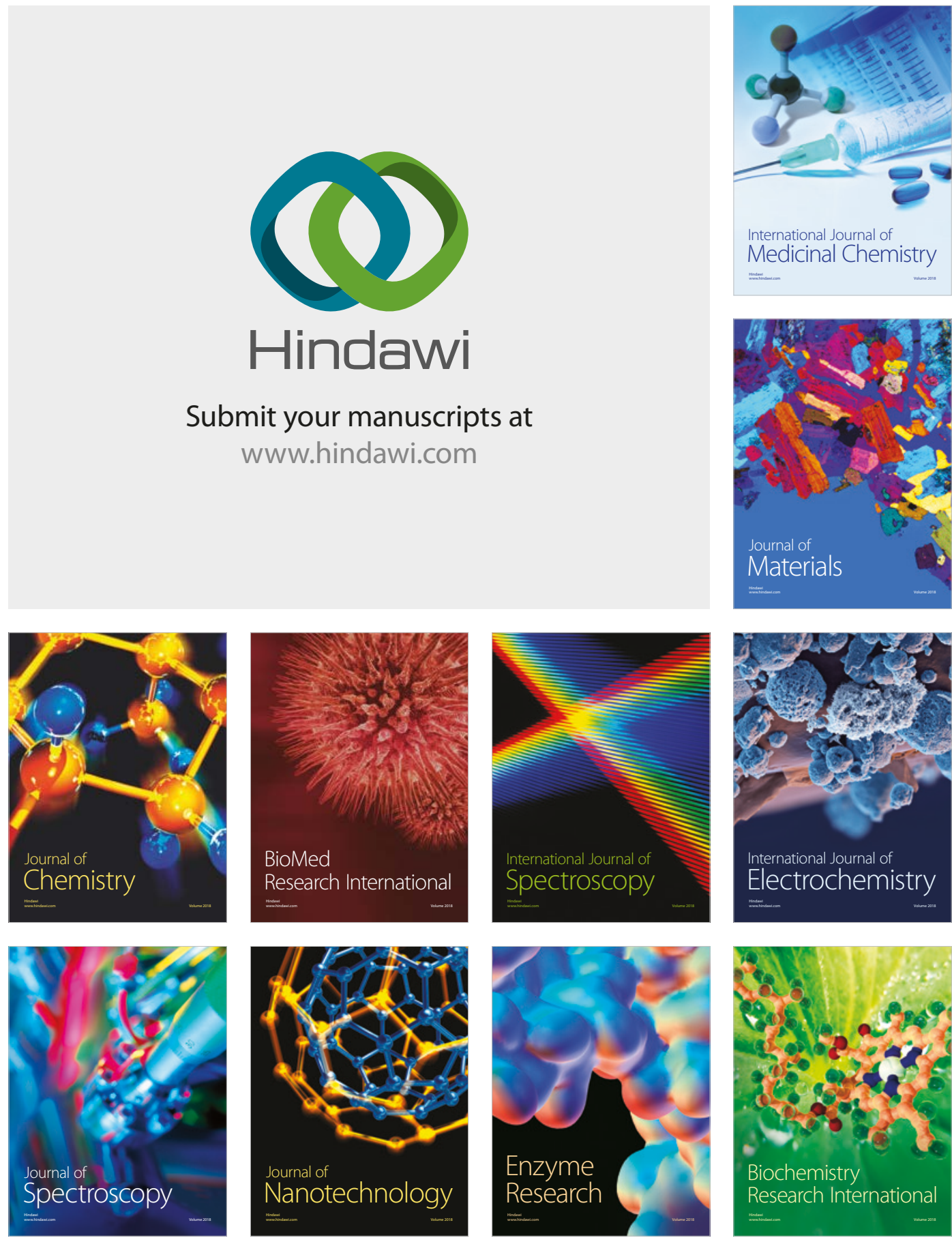
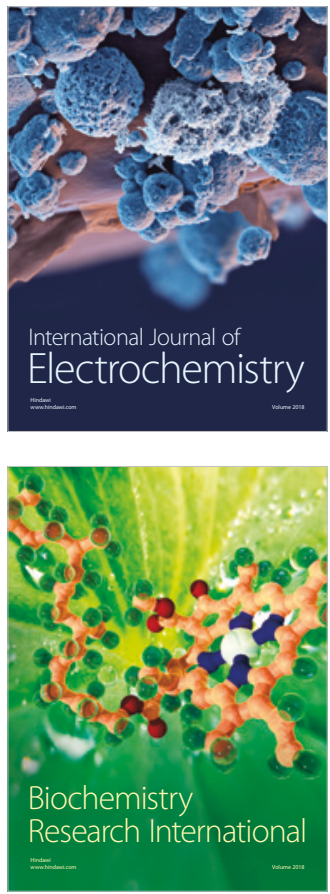